\title{
Early medical abortion using low-dose mifepristone followed by buccal misoprostol: a large Australian observational study
}

Philip Goldstone MB BS(Hons) Medical Directo

Jill Michelson

RN, RM

MBA(HealthAdmin) Risk and Quality Consultant

Eve Williamson

BPharm, PDM Business Development Consultant

Marie Stopes International Australia, Melbourne, VIC.

Philip.Goldstone@ mariestopes.org.au

MJA 2012; 197: 282-286 doi: 10.5694/mjal2.10297 tations up to 63 days, with complete abortion generally occurring in $93 \%-$ $98 \%$ of cases, incomplete abortion in $1.1 \%-4.2 \%$, and ongoing pregnancy in $0.2 \%-2.7 \% .^{3-10}$

Since its first registration in France in 1988, mifepristone has been registered in around 50 countries and is on the World Health Organization list of essential medicines. ${ }^{11}$ Mifepristone is currently an unapproved medicine in Australia, but can be accessed under the Authorised Prescriber provisions of the Therapeutic Goods Act 1989. ${ }^{12}$ Results for Authorised Prescriber use of mifepristone in Australia ${ }^{12-14}$ have been similar to those reported for other countries. ${ }^{1}$

The not-for-profit, non-governmental organisation Marie Stopes International Australia (MSIA) started Authorised Prescriber use of mifepristone-buccal misoprostol for EMA in Australia in 2009. This article describes outcomes from the use of this regimen in 13345 EMAs.

\section{Methods}

\section{Study information}

This retrospective multicentre observational study was conducted at 15 MSIA clinics in New South Wales, Victoria, Queensland, Western Australia and Editorialp $\mathbf{2 5 7}$ the Australian Capital Territory.

Abstract

Objective: To describe the use of mifepristone in combination with buccal misoprostol in women undergoing an early medical abortion (EMA) in Australia.

Design, setting and participants: Retrospective, observational study of 13345 EMAs (gestational age $\leqslant 63$ days) conducted at 15 Marie Stopes International Australia clinics between 1 September 2009 and 31 August 2011.

Intervention: Oral mifepristone $200 \mathrm{mg}$, administered at the clinic, followed 24-48 hours later by buccal misoprostol $800 \mathrm{\mu g}$, self-administered at home.

Main outcome measure: Failure rate (proportion of women with an incomplete abortion requiring surgical aspiration or a continuing pregnancy).

Results: Pregnancy termination follow-up information was available for $83.4 \%$ (11 155/13 376) of EMAs. From the patient demographic database, the EMA failure rate was 3.5\% (465/13345). Of these, most (382; $2.9 \%$ of total) were incomplete abortions requiring surgical aspiration, and 83 ( $0.6 \%$ of total) were continuing pregnancies. Haemorrhage $(16 ; 0.1 \%)$ and known or suspected infection (25; 0.2\%) were infrequent. One woman, who did not seek follow-up despite signs of infection, died from sepsis (<0.01\%). In 6755 EMAs with clinic follow-up from April 2010 to August 2011, 6381 women participated in a survey. Most reported medium or heavy bleeding and moderate or severe pain/cramps; most also reported that bleeding, pain/cramps and their overall experience were as expected or better than expected.

Conclusions: Mifepristone, with buccal misoprostol self-administered at home, for EMA up to 63 days of gestation had a low failure rate, was well accepted, and provided an effective treatment option with a favourable safety profile for women seeking an abortion in Australia.

Women undergoing a medical abortion between 1 September 2009 and 31 August 2011 were retrospectively identified and data were collected from their patient records. The study was conducted in accordance with the Declaration of Helsinki. All women provided written informed consent for treatment. The Authorised Prescriber protocol and, retrospectively, publication of the data were approved by the Queensland Clinical Trials Network Human Research Ethics Committee. The study was retrospectively registered in the Australian New Zealand Clinical Trials Registry (ACTRN12611001051932).

\section{Eligibility criteria}

Women eligible for EMA had gestations of up to 63 days, as confirmed by ultrasound, and met the relevant state or territory's legal requirements for pregnancy termination.

Exclusion criteria included known or suspected ectopic pregnancy; concomitant administration of anticoagulants or corticosteroids; adrenal failure, inherited porphyria or haemorrhagic disorder; allergy to mifepristone and/or misoprostol; intrauterine device in situ; and pelvic infection. Unless they declined, women were screened for Chlamydia trachomatis and, based on a risk assessment, screened for other sexually transmitted infections. Women were not excluded based on their proximity to emergency medical care.

\section{Treatment regimen}

The mifepristone-buccal misoprostol regimen selected was based on the peer-reviewed literature, extensive experience of Marie Stopes International with mifepristone in the United Kingdom, and MSIA's 2-year experience using methotrexate-buccal misoprostol for medical abortion to 49 days of gestation. Mifepristone $200 \mathrm{mg}$ (Linepharma) was administered orally at the clinic, followed 2448 hours later by buccal misoprostol $800 \mu \mathrm{g}$ (Pfizer), self-administered at home. Buccal administration involved placing four $200 \mu \mathrm{g}$ tablets between 
the cheek and gum for at least 30 minutes; any undissolved residue was then swallowed. If women had no bleeding within 24 hours of misoprostol administration, they were instructed to contact the clinic and return, preferably within the next 24 hours, for a second dose of misoprostol $800 \mu \mathrm{g}$. Women were informed of the signs and symptoms of possible complications (including infection) and what to do if complications occurred. Women were strongly encouraged to have a support person present throughout the process and to attend a mandatory clinic visit about 2 weeks after their initial treatment to confirm pregnancy termination and exclude complications. All women had access to an MSIA-funded, Melbourne-based, 24-hour after-care, nurse telephone service; women with issues of concern were referred to an MSIA clinic or their local medical services. Prophylactic antibiotics were not routinely prescribed except for women considered at high risk of infection. Oral analgesics (paracetamol with codeine or ibuprofen with or without codeine) were recommended. Rhesus status was determined and rhesus D-negative women were administered rhesus D immunoglobulin (250 IU) at the time of mifepristone administration.

\section{Assessments}

Information on EMA outcomes and complications was collected, including incomplete abortion requiring surgical aspiration; ongoing pregnancy; bleeding; and infection. Pregnancy termination was confirmed using a combination of ultrasound, urine pregnancy test, serum $\beta$-human chorionic gonadotropin levels, patient history, and symptoms. At the followup visit, women were asked to complete a seven-item questionnaire on pain, bleeding and their overall experience; however, not all women completed the questionnaire or all questions. Questionnaire responses from April 2010 to August 2011 were analysed (the questionnaire was expanded in April 2010; data before this date were excluded).

\section{Data collection and analysis}

Patient demographic, follow-up and complications data were collected in three separate databases, with slight variances in the total numbers of EMAs. Patient disposition was summarised using frequency counts for the number of women undergoing an EMA, the number of EMAs conducted, and the proportion of women with a follow-up visit. Demographic variables were summarised using descriptive statistics, including mean, standard deviation and range for continuous variables, and frequencies and percentages for categorical variables. The number and type of complications were summarised using frequency counts and proportions. The failure rate was defined as the proportion of women with a treatment outcome of incomplete abortion requiring surgical aspiration or of continuing pregnancy. Statistical analyses were conducted using TIBCO Spotfire $\mathrm{S}+$, version 8.2 (TIBCO Software Inc).

\section{Results}

\section{Demographics}

Between 1 September 2009 and 31 August 2011, 13345 EMAs were conducted at 15 MSIA clinics using the mifepristone-buccal misoprostol regimen (Box 1). Of the 12968 women who had an EMA, 362 women $(2.8 \%)$ had more than one EMA (Box 1). The mean age of the women was 28.4 years (SD, 6.8 years) (Box 1) and the mean length of gestation was 6.3 weeks (SD, 0.9 weeks) (Box 2).

\section{Follow-up}

For most EMAs (11 155/13 376, $83.4 \%$; Box 3 ), follow-up information was obtained, primarily via a clinic visit, to confirm pregnancy termination. During the study, an extra 31 EMAs were recorded in the follow-up database (13 376) than in the patient demographics database (13 345; 0.2\% variance). This discrepancy may be related to duplication of patient records in the follow-up database and carryover of follow-up records from a previously used treatment protocol for EMA.

\section{Complications and failure rate}

The overall complication rate was low (519/13345, 3.9\%; Box 4). The EMA failure rate was $3.5 \%$ (465). Incomplete abortion requiring surgical aspi-

1 Demographic data for women undergoing early medical abortion (EMA) with mifepristone-buccal misoprostol

Characteristic

\begin{tabular}{lc}
\hline Age, years & $28.4(6.75)$ \\
Mean (SD) & $14-49$ \\
Range & 13345 \\
Total no. of EMAs & 12968 \\
No. of women undergoing an EMA & \\
No. of EMAs per woman & $12606(97.21 \%)$ \\
1 & $348(2.68 \%)$ \\
2 & $13(0.10 \%)$ \\
3 & $1(<0.01 \%)$ \\
4 & \\
No. of EMAs per age category* & $8(0.06 \%)$ \\
14 years & $931(6.98 \%)$ \\
$15-19$ years & $3546(26.57 \%)$ \\
$20-24$ years & $3559(26.67 \%)$ \\
$25-29$ years & $2543(19.06 \%)$ \\
$30-34$ years & $1797(13.47 \%)$ \\
$35-39$ years & $855(6.41 \%)$ \\
$40-44$ years & $105(0.79 \%)$ \\
$45-49$ years &
\end{tabular}

ration occurred in $382(2.9 \%)$ cases and continuing pregnancy in 83 $(0.6 \%)$ cases. There were 16 cases of haemorrhage $(0.1 \%)$. There were four cases $(0.03 \%)$ of known and 21 cases $(0.2 \%)$ of suspected infection, including one death from sepsis $(<0.01 \%)$. This woman suffered fever and flulike symptoms about 6 days after taking mifepristone, but unfortunately did not seek medical advice, despite urging from family members. She died 9 days after taking mifepristone. Group A streptococcus (Streptococcus pyogenes) was identified from a vaginal swab and blood culture.

\begin{tabular}{|c|c|}
\hline \multicolumn{2}{|c|}{$\begin{array}{l}2 \text { Length of gestation for women } \\
\text { undergoing early medical abortion } \\
\text { (EMA) with mifepristone-buccal } \\
\text { misoprostol ( } n=13345)\end{array}$} \\
\hline Gestation & No. of EMAs* \\
\hline \multicolumn{2}{|l|}{ Length, weeks } \\
\hline Mean (SD) & $6.3(0.93)$ \\
\hline Range & $5-9$ \\
\hline \multicolumn{2}{|c|}{ Gestation category } \\
\hline 5 weeks & 2713 (20.33\%) \\
\hline 6 weeks & $5042(37.78 \%)$ \\
\hline 7 weeks & $4100(30.72 \%)$ \\
\hline 8 weeks & 1441 (10.80\%) \\
\hline 9 weeks & 49 (0.37\%) \\
\hline
\end{tabular}


3 Follow-up contact for women undergoing early medical abortion (EMA) with mifepristone-buccal misoprostol

\begin{tabular}{lccc} 
& No. of EMAs & Follow-up contact & No follow-up contact \\
\hline Total & $13376 *$ & $11155(83.40 \%)^{\dagger}$ & $2221(16.60 \%)$ \\
State/territory & & & \\
ACT & 629 & $541(86.01 \%)$ & $88(13.99 \%)$ \\
NSW & 5178 & $4536(87.60 \%)$ & $642(12.40 \%)$ \\
Qld & 2887 & $2441(84.55 \%)$ & $446(15.45 \%)$ \\
Vic & 2911 & $2154(74.00 \%)$ & $757(26.00 \%)$ \\
WA & 1771 & $1483(83.74 \%)$ & $288(16.26 \%)$ \\
\hline
\end{tabular}

$A C T=$ Australian Capital Territory. NSW $=$ New South Wales. Qld $=$ Queensland. Vic $=$ Victoria. WA $=$ Western Australia. $*$ During the study, an extra 31 EMAs were recorded in the follow-up database (13376) than in the patient demographics database (13345; $0.2 \%$ variance). This discrepancy may be related to duplication of patient records in the follow-up database and carryover of follow-up records from a previously used treatment protocol for EMA. † Follow-up contact was made via a clinic visit or a telephone call. The use of different databases for recording those who had any follow-up (11 155/13 376; clinic or phone) and those who specifically had a clinic visit (8955/ 13345) precludes a precise breakdown of the type of follow-up contact (clinic or phone). As 8955 women were known to have a clinic-based follow-up, about 2175 women may have had a phonebased follow-up (ie, assuming $83.4 \%$ of 13345 patients had any type of follow-up).

\section{Feedback on the EMA method}

From April 2010 to August 2011, 10093 EMAs were conducted. In 6755 of these EMAs, women attended follow-up and were asked to complete a seven-item questionnaire; if a woman had more than one EMA, she was asked to fill out a questionnaire for each EMA. Responses were available from 6381 women. Almost all of the women reported medium or heavy bleeding (5914/6330, 93.4\%) and the majority reported moderate or severe pain/ cramps (5001/6381, 78.4\%) (Box 5, A

\section{Reported complications for women undergoing early medical abortion (EMA) with mifepristone-buccal misoprostol}

No. of

complications*

Total no. of EMAs

13345

Complications ${ }^{\dagger}$ $519(3.89 \%)$

Type of complication ${ }^{\dagger}$

Incomplete abortion requiring surgical aspiration

Continuing pregnancy ${ }^{\ddagger}$

$382(2.86 \%)$

Haemorrhage with transfusion

$83(0.62 \%)$

$11(0.08 \%)$

$5(0.04 \%)$

$21(0.16 \%)$

Suspected infection

$4(0.03 \%)$

Known infection

$5(0.04 \%)$

Pain requiring hospital treatment

$2(0.01 \%)$

administration

Drug reaction to misoprostol

Death $^{\xi}$

$1(<0.01 \%)$

Other (not specified) and B). Most women said the bleeding $(5226 / 6233,83.8 \%)$, pain/cramps (4829/6340, 76.2\%), and overall experience $(5660 / 6265,90.3 \%)$ were as expected or better than expected (Box 5, C-E). Most women reported that they would choose the EMA method again $(4939 / 6335,78.0 \%)$ and would recommend it to a friend $(4770 / 5196$, 91.8\%) (Box 5, F and G).

\section{Discussion}

This is the first large-scale Australian study of mifepristone for EMA up to 63 days of gestation. Clinic-administered mifepristone $(200 \mathrm{mg})$ in combination with home-administered buccal misoprostol $(800 \mu \mathrm{g})$ was associated with a low failure rate, low rates of haemorrhage and infection, and was well tolerated.

The low failure rate (3.5\%) with mifepristone in our study was similar to that reported for mifepristone in randomised controlled trials (failure rates, $2 \%-7 \%)^{3-10}$ and in other Australian Authorised Prescriber Schemes (failure rates, $2.9 \%-$ $5.6 \%) .{ }^{13,14}$ Notably, the rate of surgical intervention for incomplete abortion declined from $4.3 \%$ during the first 3-month period of our study to $1.6 \%-2.6 \%$ during subsequent 3month periods. We propose that as clinic staff gained experience with the EMA method, they felt more comfortable with expectant management rather than proceeding directly to surgical intervention.

In our study, there were four cases of known infection reported $(0.03 \%)$, including one death from sepsis.

* Figures represent number of complications, except that relating to total number of EMAs. Proportion of complications calculated based on the total number of EMAs (13 345), not the number of EMAs with follow-up data (11 155); the number and type of complications could not be calculated for EMAs without follow-up data. $\neq 82$ of the 83 women had surgical

intervention; one woman chose to continue the pregnancy and delivered

a healthy baby. §One woman died from sepsis as a result of group A

Streptococcal (Streptococcus pyogenes) infection.

Infection following medical termination of pregnancy is reported to occur in $0.02 \%-0.92 \%$ of cases. ${ }^{15,16}$ Death resulting from infection and fatal toxic shock after medical abortion with mifepristone is a known, but very rare, risk $(1.1 / 100000){ }^{15}$ Although the $\mathrm{RCOG}^{2}$ recommends universal prophylactic antibiotics effective against C. trachomatis and anaerobes to reduce the risk of infection after medical abortion, the $\mathrm{WHO}^{17}$ does not. At MSIA, all women are screened for $C$. trachomatis, unless they decline, and for other STIs based on a risk assessment. MSIA has upgraded its warnings to women regarding serious infections and also now provides an opt-in SMS service that reminds women 3-5 days after their visit about the after-care phone number and signs and symptoms of complications.

The regimen we used is similar to those recommended by the $\mathrm{WHO}^{17}$ and the RCOG, ${ }^{2}$ with buccal administration of misoprostol. Vaginal misoprostol has been associated with deaths from toxic shock syndrome in the United States ${ }^{18}$ and some women may prefer buccal, rather than vaginal, administration. In addition, oral misoprostol is not sufficiently effective beyond 49 days of gestation. ${ }^{1,2,17}$ Randomised controlled trials have shown mifepristone with buccal misoprostol to have efficacy similar to or greater than mifepristone with vaginal ${ }^{7}$ or oral $^{19}$ misoprostol for terminating pregnancies up to 56 and 63 days, respectively. Our study provides further support for using $200 \mathrm{mg}$ of mifepristone, which is lower than the $600 \mathrm{mg}$ dose approved in the US and Europe. ${ }^{1}$ Randomised controlled trials directly comparing the two mifepristone doses have shown similar efficacy for the $200 \mathrm{mg}$ and $600 \mathrm{mg}$ doses. ${ }^{3,6}$ A Cochrane meta-analysis also found no difference in failure rates between the $200 \mathrm{mg}$ and $600 \mathrm{mg}$ doses. $^{20}$

In our study, all women were informed of the need for mandatory follow-up to confirm pregnancy termination; contact with most women was achieved. Those who did not attend the clinic were telephoned and, if telephone contact was not possible, a registered letter was sent (the letter included a pregnancy test kit to facilitate home-testing). Despite these efforts, follow-up information was 
missing for around $17 \%$ of women. The loss to follow-up in our real-world setting of mifepristone use was higher than that observed in rigorous clinical trial settings $(1.7 \%-8.6 \%) .{ }^{3-5,8,9,21}$ As might be expected in clinical practice, not all patients may return for followup, even if instructed to attend a follow-up visit to assess treatment outcome. Given the emotional issues surrounding abortion, women who felt the abortion was complete and had no physical complications may have been reluctant to return to the clinic. While this is of concern, a recent study using early telephone follow-up after medical abortion to determine the need for further evaluation found that women who initially had not required evaluation returned to the clinic of their own accord if they experienced problems, such as bleeding or continuing pregnancy. ${ }^{21}$ This finding suggests that women who experience adverse effects or feel that the abortion is not complete are likely to seek appropriate medical care. Indeed, in our study, about one-third of women did call the MSIA after-care service about issues such as pain or bleeding. While many women required reassurance only, some callers were referred to the clinic or a hospital for further assessment. In addition, it is possible that some women received follow-up care elsewhere. Not all women with complications, however, will decide to seek follow-up care and, in very rare cases (eg, one out of 13345 cases in our study), this can have serious consequences. Understandably, such cases can raise concerns about the safety of home-based versus clinicbased administration of misoprostol. Although misoprostol could be administered in a clinic under medical supervision, home-based administration of misoprostol has been associated with a low complication rate, is preferred by women, and is common practice in France and the US. ${ }^{1,22}$ Further, clinic-based administration of misoprostol would not necessarily enhance outcomes as medical abortion complications can occur hours or days after misoprostol is administered.

Overall, there was a high level of satisfaction with the mifepristonebuccal misoprostol regimen used, with most women who completed the study questionnaire reporting the bleeding, pain/cramps, and overall experience to be as they had expected or better than expected. Satisfaction with the EMA method used in our study was similar to that reported in other studies. ${ }^{1,23,24}$ Notably, there has been a significant uptake of medical abortion in our clinics, with about a third of women requesting an abortion up to 63 days of gestation opting for the medical method (data not shown).

Our mifepristone-buccal misoprostol EMA method could be used outside a hospital setting. The diagnosis and initiation of treatment (mifepristone) can be conducted in a clinic and the second part of the treatment (misoprostol) can be carried out by the woman at home, with 24-hour access to after-care services to manage any issues. This arrangement, along with the 24-48-hour window for misoprostol administration, allows the abortion process to take place in the privacy of the woman's home and at the time of her choosing, factors known to be of importance to women undergoing an abortion., 1,23,24 The clinic- and home-based nature of the mifepristone-buccal misoprostol regimen makes it feasible for use in regional centres of Australia. These centres may have smaller hospitals than urban areas, but have the facilities and staff to manage spontaneous miscarriage. ${ }^{24}$ The MSIA clinics are located in major metropolitan areas and women are provided with discharge letters. Any woman contacting the after-care service can be referred back to the MSIA clinic or to her local doctor or, if necessary, a nearby accident and emergency facility. In clinical

5 Results of patient survey on the early medical abortion method, April 2010 - August 2011
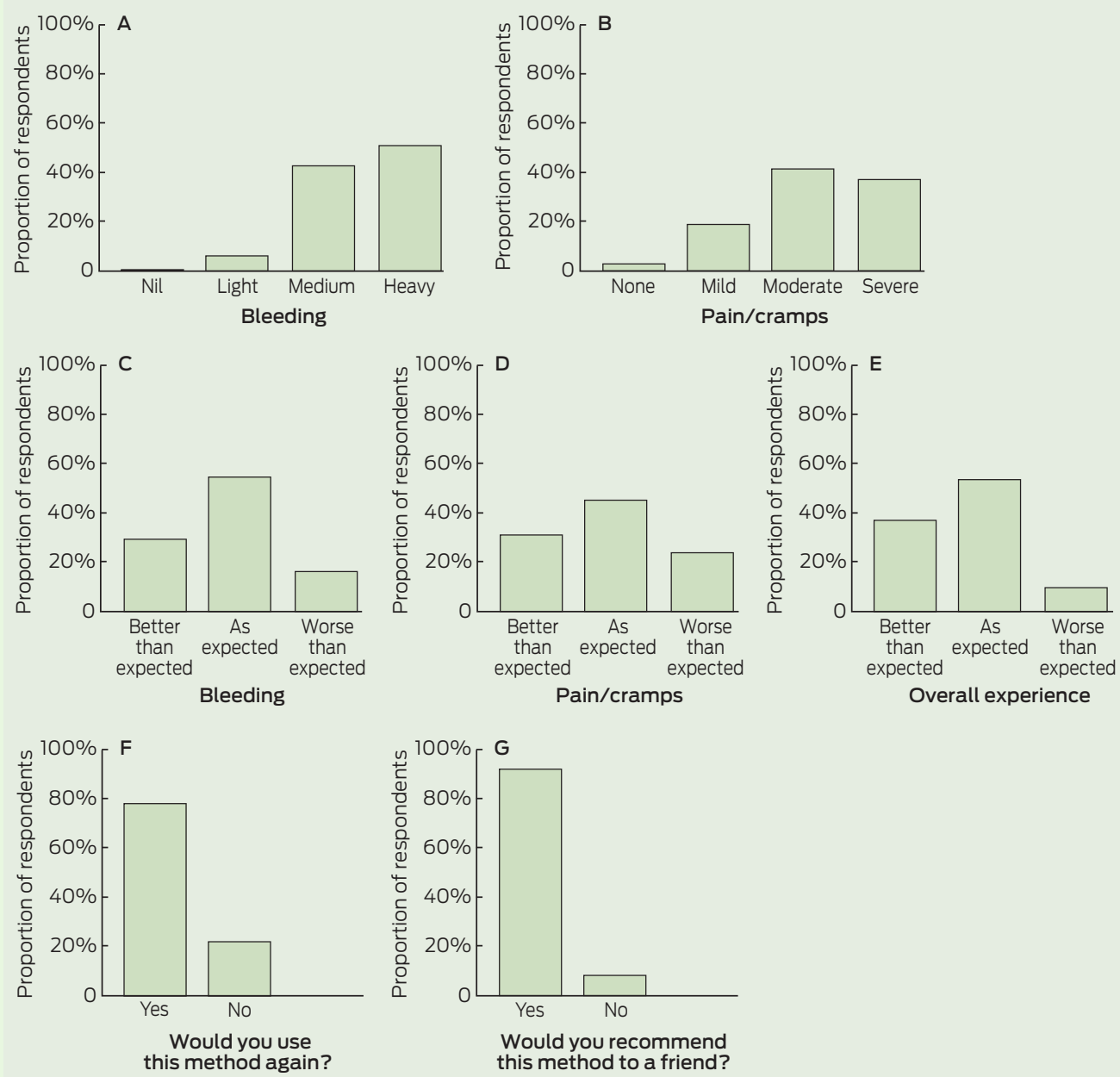

Overall experience

Women were asked (i) to describe the severity of their bleeding $(A ; n=6330)$ and pain/cramps $(B ; n=6381)$; (ii) whether the bleeding $(C ; n=6233)$, pain/cramps $(D ; n=6340)$, and overall experience $(E ; n=6265)$ were better than expected, as expected, or worse than expected; (iii) whether they would choose the method again ( $F ; n=6335$ ); and (iv) whether they would recommend the method to a friend $(G ; n=5196)$. 
practice, proximity to emergency services should be evaluated when considering the use of the mifepristonebuccal misoprostol regimen. The potential need for access to appropriate 24-hour emergency help could limit the use of this regimen in more remote areas of Australia.

The observational nature of our study allowed assessment of the mifepristone-buccal misoprostol regimen in a real-world setting. However, as discussed, one of the study limitations was the relatively high rate of loss to follow-up. As a result, it was not possible to accurately determine the complete abortion rate; instead the known failure rate was reported. In addition, the use of multiple databases for data collection led to some small discrepancies in the datasets used for the analyses (eg, different total numbers of EMAs), because of differences in how the data were collected.

In conclusion, our Australian study of 13345 EMAs has shown that mifepristone followed by buccal misoprostol has a low failure rate, including a low ongoing pregnancy rate. The regimen, which involved self-administration of misoprostol and completion of the abortion process at home, had a favourable safety profile and was well accepted by the women in this study. While the potential risk of serious infection should be kept in mind and monitored, these results indicate that the mifepristone-buccal misoprostol regimen is an effective option for Australian women seeking an abortion up to 63 days of gestation.

Acknowledgements: This study was sponsored by Marie Stopes International Australia, a not-for-profit, non-gov- ernmental organisation. The sponsor did not impose any impediment, directly or indirectly, on the publication of our results. We acknowledge the independent medical writing assistance of Justine Southby and Julie Ely of ProScribe Medical Communications, funded from an unrestricted financial grant from MSIA. We also acknowledge the contributions to the study from the Marie Stopes Study Group: Ivana Borsky, Harry Cohen, Nicole Gastaldin, Janelle Hall, Katerina Lagios, Gary Lubransky, Alex MacPherson, Kevin Pedemont, Andrew Perry, Rebecca Quake, Charles Russell-Smith, Robin Tideman, and Ying Zhou.

Competing interests: Philip Goldstone is an employee of and Jill Michelson and Eve Williamson are consultants to, Marie Stopes International Australia.

Received 14 Feb 2012, accepted 6 Aug 2012.

1 Fiala C, Gemzel-Danielsson K. Review of medical abortion using mifepristone in combination with a prostaglandin analogue. Contraception 2006; 74: 66-86.

2 Royal College of Obstetricians and Gynaecologists. The care of women requesting induced abortion. Evidence-based clinical guideline number 7. London: RCOG, 2011. http:// www.rcog.org.uk/files/rcog-corp/

Abortion\%20guideline_web_l.pdf (accessed Jun 2012)

3 World Health Organization Task Force on Postovulatory Methods of Fertility Regulation. Comparison of two doses of mifepristone in combination with misoprostol for early medical abortion: a randomised trial. BJOG 2000; 107: 524-530.

4 Bartley J, Brown A, Elton R, Baird DT. Doubleblind randomized trial of mifepristone in combination with vaginal gemeprost or misoprostol for induction of abortion up to 63 days gestation. Hum Reprod 2001; 16: 2098-2102.

5 Creinin MD, Fox MC, Teal S, et al. A randomized comparison of misoprostol 6 to 8 hours versus 24 hours after mifepristone for abortion. Obstet Gynecol 2004; 103: 851-859.

6 McKinley C, Thong KJ, Baird DT. The effect of dose of mifepristone and gestation on the efficacy of medical abortion with mifepristone and misoprostol. Hum Reprod 1993; 8: 1502-1505.

7 Middleton T, SchaffE, Fielding SL, et al. Randomized trial of mifepristone and buccal or vaginal misoprostol for abortion through 56 days of last menstrual period. Contraception 2005; 72 328-332.

8 SchaffEA, Fielding SL, Westhoff C. Randomized trial of oral versus vaginal misoprostol 2 days after mifepristone $200 \mathrm{mg}$ for abortion up to 63 days of pregnancy. Contraception 2002; 66: 247-250.
9 Schaff EA, Fielding SL, WesthoffC, et al. Vaginal misoprostol administered 1, 2, or 3 days after mifepristone for early medical abortion: a randomized trial. JAMA 2000; 284: 1948-1953.

10 Tang OS, Chan CC, $\mathrm{Ng} \mathrm{EH}$, et al. A prospective, randomized, placebo-controlled trial on the use of mifepristone with sublingual or vaginal misoprostol for medical abortions of less than 9 weeks gestation. Hum Reprod 2003; 18 : 2315-2318.

11 Gibson L. WHO puts abortifacients on its essential drug list. BMJ 2005; 331: 68.

12 de Costa CM, Russell DB, de Costa NR, et al. Early medical abortion in Cairns, Oueensland: July 2006 - April 2007. Med J Aust 2007; 187: 171-173.

13 de Costa CM. Use of mifepristone for medical abortion in Australia, 2006-2009. Med J Aust 2011; 194: 206-207.

14 Mulligan E, Messenger $\mathrm{H}$. Mifepristone in South Australia - the first 1343 tablets. Aust Fam Physician 2011; 40: 342-345.

15 Henderson JT, Hwang AC, Harper CC, Stewart FH. Safety of mifepristone abortions in clinical use. Contraception 2005; 72: 175-178.

16 Shannon C, Brothers LP, Philip NM, Winikoff B. Infection after medical abortion: A review of the literature. Contraception 2004; 70: 183-190.

17 World Health Organization. Safe abortion: technical and policy guidance for health systems. 2nd ed. Geneva: WHO, 2012.

18 Fischer M, Bhatnagar J, Guarner J, et al. Fatal toxic shock syndrome sssociated with Clostridium sordellii after medical abortion. New Engl J Med 2005: 353: 2352-2360.

19 Winikoff B, Dzuba IG, Creinin MD, et al. Two distinct oral routes of misoprostol in mifepristone medical abortion: a randomized controlled trial. Obstet Gynecol 2008; 112: 1303-1310.

20 Kulier R, Kapp N, Gulmezoglu AM, et al. Medical methods for first trimester abortion. Cochrane Database Syst Rev 2011; (11): CD002855. DOl: 10.1002/14651858.CD002855.pub4

21 Perriera LK, Reeves MF, Chen BA, et al. Feasibility of telephone follow-up after medical abortion. Contraception 2010; 81: 143-149.

22 Ngo TD, Park MH, Shakur H, Free C. Comparative effectiveness, safety and acceptability of medical abortion at home and in a clinic: a systematic review. Bull World Health Organ 2011; 89 : 360-370

23 Fiala C, Winikoff B, Helstrom L, et al. Acceptability of home-use of misoprostol in medical abortion. Contraception 2004; 70: 387-392.

24 de Costa CM. Medical abortion for Australian women: it's time. Med J Aust 2005; 183: 378-380. 